taken of the change of resistance under pressure of a "therlo" wire, and in order to determine the pressure to within one megabar the resistance change was measured with a type of Wheatstone bridge having no movable contacts.

The $P$ - $\Delta v$ graph for gold, copper, brass, silver, aluminum, and calcite, like that of steel, is sensibly linear; but the graph for zinc, tin, cadmium, lead, tin-bismuth alloy, quartz, bismuth, and sodium chloride shows an appreciable curvature, ${ }^{1}$ thus indicating for those substances a measurable decrease of compressibility with increasing pressure.

A comparison was made of the compressibility of two alloys with that of their components. In the case of a simple mixture such as the tinbismuth alloy, the measurements indicate that the compressibility of mixtures whose other properties, such as specific volume, electrical conductivity, and specific heat, are approximately linear functions of the composition, is related in the same simple way to the compressibilities of the separate components. On the other hand, the compressibility of alloys of the class to which brass belongs is much lower than the sum of the individual compressibilities.

WASAINCTON, D. C.

[CONTRIBUTION FROM Kent Chamical Laboratory, University of Chicago.]

\title{
A STUDY OF THE EMANATION METHOD OF DETERMINING THORIUM.
}

BY G. H. CARTIEDGE.

Received September 9, 1918.

The well-known emanation method is the best one for estimating small amounts of radium. But thorium cannot be determined similarly because of the very short period of its amanation. However, it has been proposed to modify the method by circulating air continuously through the solution of the thorium preparation and through the ionization chamber noting the activity when the system has reached a steady state.

Although there are a few references in the literature to such a method, ${ }^{2}$ so far as the author is aware no one except Joly ${ }^{3}$ has ever used it for quantitative purposes; and in that work it was applied only to the analysis of ordinary rocks containing thorium to the extent of about $1 \mathrm{O}^{-5} \mathrm{~g}$. per gram of rock.

In view of the great practical value of a simple method of estimating thorium without the necessity of separating it from the other rare earths generally occurring with it in nature, and of the importance of such a

1 The measurements on silica glass were not extended far enough to determine satisfactorily the amount of its change of compressibility.

2 Soddy, "Chemistry of Radioelements."

${ }^{3}$ Joly, Phil. Mag., 17, 760 (1909). 
radio-chemical method of analysis in the solution of certain problems of purely scientific interest, it was suggested by Professor McCoy that an investigation be undertaken to ascertain what reliance can be placed in the thorium emanation method, how generally it can be employed, and especially to identify the sources of error and to determine the character of each as far as possible.

By way of anticipation it may be stated that the method described in this paper can be employed satisfactorily and conveniently wherever one can get the sample into complete solution, and that the measurements can be made with an accuracy equal to that usually obtainable

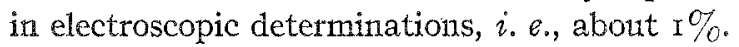

It is, of course, obvious that the emanation method is in reality a direct determination only of thorium $\mathrm{X}$, the first part of the thorium series being as follows:

$\begin{array}{ccccccc}\text { Substance. } & \text { Th. } & \text { Ms I. } & \text { Ms II. } & \text { Rt. } & \text { ThX. } & \text { Th Em. } \\ \text { Period................. } & 1.8 \times 10^{10} & 5.5 & 6.2 & 737 & 3.64 & 54 \text { sec. } \\ & \text { years } & \text { years } & \text { hours } & \text { days } & \text { days } & \\ \text { Chemical analog or isotope } & & \text { Ra } & \text { La } & \text { Th } & \text { Ra } & \text { Ra Em }\end{array}$

The first object of this investigation was, therefore, an attempt to perfect a method of determining the thorium content of pure thorium nitrate solutions by means of the emanation. When this method had been perfected it was then applied, first, to the radioactive analysis of thorium minerals and, second, to the determination of the $\gamma$-ray activity of thorium $\mathrm{D}$ as described in the following paper.

The gold-leaf electroscope used in this work and shown in Fig. I, was of the same general design as those which have been in use in this laboratory for several years. It need not be completely described here. The only alteration made for this work consists in having the standard to which the leaf is attached terminate $4 \mathrm{~cm}$. below the amber plug $\mathrm{P}$ in a double wedge-shaped spring $S$; the electrode $\vec{E}$ of the ionization chamber makes a sliding contact with this spring.

When working with samples

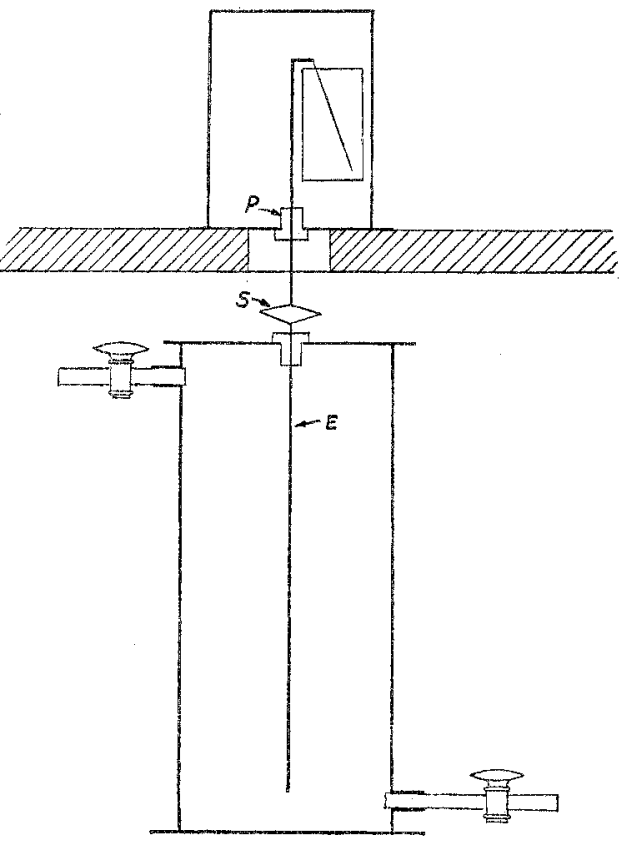

Fig. $Y$. 
containing thorium $\mathrm{X}$ equivalent to $0.2 \mathrm{~g}$. thorium or more, it is impossible to make more than 3 analyses a day with one ionization chamber, owing to the accumulation of the active deposit of thorium emanation. For this reason interchangeable ionization chambers have been constructed so

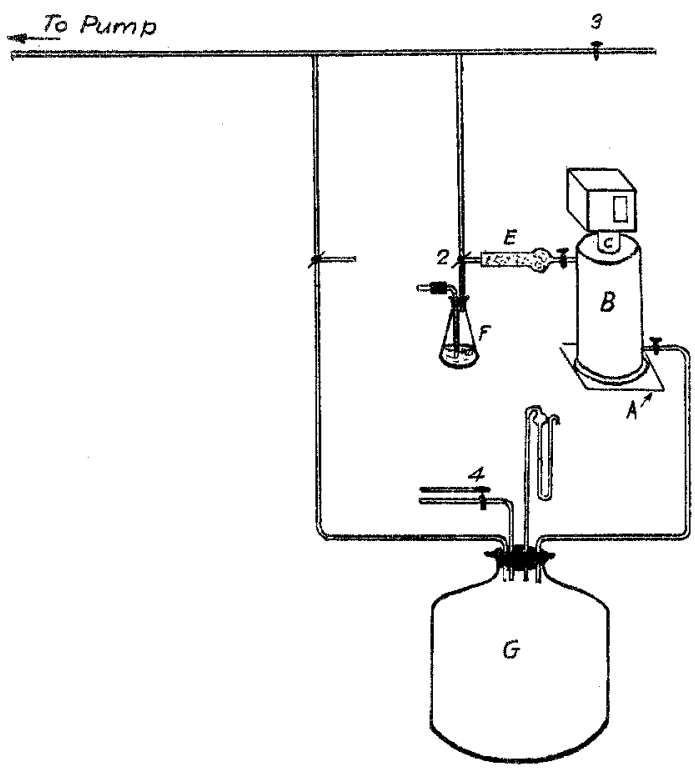

Fig. 2 . that it is not necessary to wait for the decay of the active deposit before using the electroscope for another determination. The chambers are of brass "telescope tubing" $\mathrm{r} 4 \mathrm{~cm}$. in diameter, and $31 \mathrm{~cm}$. in height. A brass rod 0.4 by $25 \mathrm{~cm}$. is fastened at the axis of the cylinder by means of an amber plug and projects $0.6 \mathrm{~cm}$. above the top of the plug. As an electrostatic screen, a brass collar, $\mathrm{C}$, in Fig. 2 , of about ro $\mathrm{cm}$. diameter and long enough to clear the ionization chamber is attached to the shelf carrying the electroscope. A slot in the collar allows the amber plug and electrode extension to pass, the slot being closed by a movable screen after the ionization chamber is in place. The brass plate $A$, the protecting collar, and the case of the electroscope are all grounded.

Two different methods of circulating air carrying the thorium emanation through the ionization chamber were devised and thoroughly studied. In the first method a motor-driven diaphragm pump, designed and constructed for this work was used. It had no stuffing boxes and was leakproof. This method gave fair results, but it was abandoned in favor of a second and more satisfactory method.

In the second method free air was drawn at a constant rate through the solution and ionization chamber by means of a constant pressure aspirator. In this case the air leaving the electroscope did not pass through the thorium solution again.

The arrangement of the spirator and its accessories is shown in Fig. 2 , in which $I$ and 2 are three-way glass stopcocks, while 3 and 4 are twoway cocks. $G$ is a ro-gallon bottle for equalizing the pressure, and is connected with a manometer of sufficient size to allow a difference of pressure of $r_{5} \mathrm{~cm}$, olive oil. Stopcock 4 allows of the admission of vari- 
able quantities of air to the system in order to maintain a constant pressure difference of $\mathrm{x} 2 \mathrm{~cm}$. of olive oil, as indicated by the manometer. A calcium chloride tube $\mathrm{E}$ is inserted between the solution in $\mathrm{F}$ and the ionization chamber B.

Air is drawn through the solution in $\mathrm{F}$ as fast as is possible without danger of mechanical loss. In order to increase the pressure difference corresponding to this optimum air current a constricted tube is attached to the inlet tube of the flask; in this way the slight but unavoidable fluctuations in the friction through the apparatus are made of relatively less importance.

The process of an activity measurement is as follows: the ionization chamber is evacuated for at least 20 minutes, keeping the gold-leaf charged during this time. Air is then drawn through the cylinder for about 5 minutes, after which the natural leak of the electroscope is taken while air is drawn through the ionization cylinder after passing through a flask containing distilled water, under the same conditions as in the activity measurements. With stopcock 2 connecting $\mathrm{E}$ and $\mathrm{F}$ open and cock 3 closed, the pressure can be most easily controlled by turning I so that there is only partial connection between the vacuum line and $G$, allowing a good vacutum to be maintained in the vacuum line. During the determination of the natural leak the solution to be tested is attacked to 3 and aspirated to remove radium emanation; this aspiration should last at least 15 minutes if the sample contains appreciable quantities of radium.

For the most accurate work the cylinder should now be evacuated again for about 5 minutes, opened up to atmospheric pressure, and the activity of a standard radium tube noted when the tube is placed in a stationary holder on the top of the electroscope. The radium tube used in these experiments contained $0.355 \mathrm{mg}$. radium element. The thorium solution is now connected as shown in the figure, with constricted tube attached, and a moderate current of air drawn through it (but not through the ionization chamber) for about ro seconds; stopcocks I and 2 are then rapidly adjusted so as to draw the emanation through the drying tube and ionization chamber, and the pressure regulated to the fixed mark on the manometer. After about 3 minutes the activity will have become constant.

The pressure must be kept very close to a fixed value; but the experimental arrangement allows of quite satisfactory control even on a water line subject to wide variations of pressure.

Since a portion of the thorium emanation will always decay before reaching the ionization chamber it is obvious that solutions to be comparable must be contained in vessels of about the same size, in order 
that the free volume between solution and chamber may be as nearly constant as possible.

Samples to be analyzed must be contained in equal volumes. This volume variation differs somewhat according to the nature of the solution, but the order of magnitude can be seen from the fact that for a particular solution of $75 \mathrm{cc}$. volume, the addition of $20 \mathrm{cc}$. water decreased the activity $13.6 \%$.

It is not generally true that the activity is exactly proportional to the thorium $\mathrm{X}$ content when solutions of moderately different concentration are compared. The relation depends considerably on the form of apparatus and its adjustment, as well as on the type of solution used, so that it has to be determined for any particular case. The following data, however, will indicate the general behavior that has been observed in this work with perfectly clear solutions of thorium or radiothorium nitrate:

$\begin{array}{ccc}\begin{array}{c}\text { Concentration. } \\ \text { In arbitrary units. }\end{array} & \begin{array}{c}\text { Activity. } \\ \text { In arbitrary utits. }\end{array} & \begin{array}{c}\text { Activity } \\ \text { Concentration. }\end{array} \\ 0.60 & 0.632 & 1.052 \\ 0.742 & 0.763 & 1.029 \\ 0.75 & 0.772 & 1.028 \\ 0.75 & 0.777 & x .034 \\ 0.80 & 0.815 & 1.019 \\ 0.80 & 0.815 & 1.019\end{array}$

It is probable that the ionization current is not the saturation current: in any case, but approaches it more nearly the smaller the amount of emanation.

In all cases examined there is proportionality between activity and concentration within experimental error if the solutions do not differ more than $8 \%$ in thorium $\mathrm{X}$ content. In practise, then, it is necessary either to use a series of standard solutions and select for each analysis one of about the same activity as that of the unknown measured, or else to plot a concentration-activity curve for the particular type of solution under investigation, reading off the concentration corresponding to any measured activity.

The activity of a solution measured by the emanation method varies greatly with alterations in the temperature. The following table summarizes the results for 3 typical solutions, when the activity was noted with the solution maintained first at one temperature and then at another, the rest of the apparatus being all the while at room temperature:

\begin{tabular}{|c|c|c|c|c|c|}
\hline $\begin{array}{l}\text { Solution. } \\
\operatorname{Th}\left(\mathrm{NO}_{3}\right)_{4} \ldots \ldots\end{array}$ & $\begin{array}{r}T_{2} \\
22^{\circ}\end{array}$ & $\begin{array}{c}\text { Activitys. } \\
0.8 \mathrm{II}\end{array}$ & $\begin{array}{l}T_{2} . \\
\text { II }^{\circ}\end{array}$ & $\begin{array}{c}\text { Activity. } \\
0.737\end{array}$ & $\begin{array}{c}a . \\
0.0084\end{array}$ \\
\hline $\mathrm{Th}\left(\mathrm{NO}_{3}\right)_{4}, \ldots \ldots \ldots \ldots \ldots \ldots$ & 21.5 & 0.909 & $\mathrm{I} 6.5$ & 0.882 & 0.0058 \\
\hline Thorianite. ............... & 27 & $1.26 I$ & 16 & I. 127 & 0.0097 \\
\hline
\end{tabular}

$a$ is the temperature coefficient defined by the equation Activity $2=$ Activity $_{1}(\mathrm{I}-a \Delta t), \Delta t$ being the decrease in temperature. 
In view of the relatively long period of radium emanation, the thorium emanation can be accurately determined even in the presence of considerable arnounts of radium, if the solutions are thoroughly aspirated to remove radium emanation before beginning a determination. Actinium might be expected to interfere, but such is not the case, except when it is present in large amount, because of the rapid decay of actinium emanation.

\section{The Determination of Thorium in Monazite Sand by the Emana- tion Method.}

From a practical standpoint, by far the most important application of the thorium emanation method is to the analysis of monazite, which mineral is almost the sole source of commercial thorium. As is well known, monazite sand is essentially a phosphate of rare earths and thorium and may also contain considerable amounts of titanium, zirconium and iron. Some quartz is also present, as a rule. The India sand used in most of our experiments contained $8.57 \%$ of $\mathrm{Th}^{\mathrm{O}} \mathrm{O}_{2}$ as determined by the method of Carney and Campbell. ${ }^{1}$ Because of the necessity of making the monazite solution with sulfuric acid, coupled with the fact that thorium $\mathrm{X}$ accompanies barium sulfate, very great difficulty is encountered in attempting to get the maximum amount of thorium $\mathrm{X}$ at the start; but after investigating a number of procedures, more or less different, a method was developed which with careful work gives at once the thorium content with an error of less than $1.5 \%$ of its value as shown by gravimetric analyses.

Of the various procedures tried, the one described below gave the most concordant results.

A sample of $2 \mathrm{~g}$. of monazite sand is digested for about 3 hours with 4-5 $\mathrm{cc}$. concd. sulfuric acid at a temperature slightly below the boiling point of the acid. Resistance glass beakers of $50 \mathrm{cc}$. capacity covered with watch glasses serve very well for the digestion, being heated on a sand bath. Ice is added to the cold sulfates and after about 30 minutes the solution, diluted to about $50 \mathrm{cc}$., is poured into roo cc. of $20 \%$ sodium hydroxide previously heated to boiling in a $14 \mathrm{~cm}$. casserole; the beaker and watch glass are washed, using a policeman and retaining in the beaker the undecomposed residue of silica, ilmenite, etc., which would produce violent bumping. The hydroxide precipitate is vigorously boiled for about 3 minutes, the sand residue in the beaker washed into the casserole, and then about $15 \mathrm{cc}$. of sodium corbonate solution boiled for a few minutes in the beaker. This solution is added to the hydroxide mixture and the beaker carefully washed out with dil. hydrochloric acid, adding the washings to the casserole. The complete mixture is now raised as nearly to the boiling point as possible without danger of loss

1 This JournaI, $36, \mathrm{II}_{34}$ (I9I4). 
through bumping, ${ }^{1}$ and is then allowed to settle for a few minutes. The hydroxide mixture is filtered through a $\mathrm{r} 2 \mathrm{~cm}$. hardened paper on a Hirsch funnel with a $9 \mathrm{~cm}$. plate, gentle suction being used in the filtration. The precipitate is washed entirely free from sulfates, but it is not necessary to transfer to the paper the last quantity of matter which adheres to the casserole.

One $\mathrm{cc}_{\mathrm{b}}$ of ro\% barium chloride solution is distributed over the surface of the casserole. The paper containing the precipitated earths is then put in the casserole and another cc. of barium chloride poured over it. After stirring up the mixture for a minute or two, $20 \mathrm{cc}$. of concd. hydrochloric acid is added. As solution proceeds, the mixture is heated cautiously, taking care to spread the acid to all parts of the dish, and thoroughly to macerate the filter paper. The acid digestion should last at least 5 minutes. The mixture is not filtered but transferred in toto to the Erlenmeyer flask in which the activity measurement is to be made, washing out the casserole in all cases with identical volumes of wash water $(60 \mathrm{cc}$.). Use of the policeman is necessary, since the shredded filter paper adheres tenaciously to the casserole. In following the scheme one must very thoroughly tear the paper to pulp during the digestion and, furthermore, it is necessary to see that the paper mass is vigorously stirred at the beginning of an activity measurement by rotating and inclining the flask while the air is bubbling through it. Once mixed, the air current takes care of the paper during the rest of the measurement. The following data illustrate the accuracy with which duplicate analyses agree:

\begin{tabular}{|c|c|c|c|}
\hline Sample. & Ratio. & $\begin{array}{l}\% \mathrm{ThO}_{2} \text { by } \\
\mathrm{E}_{1} \mathrm{~m} \text { method. }\end{array}$ & $\begin{array}{l}\% \mathrm{ThO}_{2} \text { by } \\
\text { grav. analysis. }\end{array}$ \\
\hline No. ${ }_{147-148}$ & $x .005$ & $8.6 \mathrm{I}$ & 8.57 \\
\hline No. $150-15 x$ & $x, 009$ & 8.65 & 8.57 \\
\hline No. $153^{-154}$ & I. 013 & 8.68 & 8.57 \\
\hline
\end{tabular}

These analyses were made just after the preparation of the various solutions and the accuracy is seen to be quite good.

It appears perfectly safe to state that the final procedure will give the thorium content of a sample of monazite within $1.5 \%$ of its true value. In its present development, however, the emanation method cannot replace the best gravimetric analysis for monazite, and will find its greatest value in the solution of certain problems of purely scientific interest, where pure chloride solutions are easily obtainable.

\section{Summary.}

I. A convenient apparatus has been devised for circulating an air current through a solution and ionization chamber for Th emanation measurements.

${ }^{1}$ A series of experiments was run wherein this hydroxide mixture was boiled with superheated steam for an hour. No better results were obtained by this method. 
2. The sources of error in such determinations and the factors in the emanation activity of a solution have been studied.

3. A method for preparing radiochemically complete solutions of monazite sand has been worked out so as to give an accuracy of within $1.5 \%$ total $\mathrm{ThO}_{2}$.

\section{Notes on the Gravimetric Monazite Analysis.}

I. The method of analysis proposed by Carney and Campbel1 ${ }^{1}$ makes use of ammonium perchlorate with sulfuric acid for the destruction of the filter paper and conversion of thorium pyrophosphate into $\mathrm{Th}(\mathrm{SO})_{2}$. Owing to the difficulty of obtaining ammonium perchlorate it occurred to the author to try fuming nitric acid for the purpose, and the results of the attempts were so successful that it seems worth while to record them. The precipitate of thorium pyrophosphate is sucked as free as possible from water and transferred with the paper to a $250 \mathrm{cc}$. Kjeldahl flask. I5 cc. conc. sulfuric acid is added and the mixture shaken for about two minutes, cooling, if necessary, until the flask is just a little too hot to be held in the hand. By this time the mixture will be entirely charred. $2-4$ cc. of fuming nitric acid (sp. gr. I.53) are then added and after a minute or two gradually raised to the boiling point. Occasionally as the fumes of sulfur trioxide begins to appear a second charring occurs, in which case $0.5 \mathrm{cc}$. more nitric acid poured cautiously down the neck of the flask will complete the oxidation. Boiling should be continued for about 2 minutes after the sulfur trioxide fumes begin to escape freely. At the end of this digestion the mixture will be either pure white or slightly. yellow, as in the perchlorate procedure; but no harm is done if oxides of titrogen redissolve in the acid during the cooling, thereby restoring a yellow or brown color. The entire acid digestion can be carried out in less than half the time required for a perchlorate digestion, and no frothing will occur if care is taken to have the paper sucked free of excess water and yet not so dry that insufficient heat to char the paper will be generated on adding the sulfuric acid.

2. Although the directions for Carney and Campbell's method call for a filtration of the original monazite solution before it is diluted to the mark, it is known to be the practice of some analysts to introduce the fine, white turbidity along with the solution, rejecting only the undecomposed residue. In order to emphasize the error so introduced in the analysis, the author wishes to record some experiments concerning the identity of this turbidity. A $2.5 \mathrm{~g}$. sample of sand was digested and dissolved in ice water, and after long standing filtered. After thorough washing, the paper was burned and the residue weighed, treated with hydrofluoric and sulfuric acids, ignited, again weighed and fused with potassium hydrogen sulfate. The solution from this fusion was tested

: THIS JOURNAL, 36, II34 (I9I4). 
for titanium and thorium; titanium was found, but no thorium. The weighings showed that the residue was composed of silica and titanium in some form, and in about equal amounts in this particular case. Furthermore, analyses made on filtered and unfiltered solutions showed that a large part of the titanium residue appears in the final thorium dioxide thus giving an erroneously high value.

ChICAGo, Iri.

[Contribution from Kent Chemical laboratory, Univarsity of Chicago.]

THE GAMMA RAY ACTIVITY OF THORIUM D.

by Herbert N. McCoy and G. H. Carthede.

Received September 9, 1918.

Eve, ${ }^{1}$ and McCoy and Henderson ${ }^{2}$ have independently determined the quantity of thorium, in equilibrium with its products, which has the same $\gamma$ activity as one $\mathrm{g}$. radium in equilibrium. Their results were $6.9 \times 10^{6} \mathrm{~g}$. and $6.85 \times 10^{6} \mathrm{~g}$., respectively. Furthermore, McCoy and Henderson have determined the ratio $\mathrm{Ms}$ : Th, the mesothorium being expressed in terms of the weight of radium giving the same $\gamma$ activity, i. e., one mg. Ms means the amount of mesothorium whose activity equals that of one $\mathrm{mg}$. radium one month after preparation. The value found for this ratio is given as $0.52 \times 10^{-7}$. The reciprocal ratio $19.0 \times 10^{6}$ is the number of grams of thorium necessary to furnish $\mathrm{I} g$. mesothorium. Therefore, mesothorium accounts for only $6.9 / 19.0$ or $36.3 \%$ of the total $\gamma$ activity of the thorium series, although thorium $D$ is the only other thorium product which emits a $\gamma$ radiation.

It seemed, therefore, to be a matter of interest to determine independently the Th $\mathrm{D}:$ Th ratio in order to ascertain whether the combined mesothorium and thorium $\mathrm{D}$ activities would give the total $\gamma$ activity.

First Procedure.-A large quantity of radiothorium was allowed to accumulate in a very active mesothorium preparation and then separated out with aluminum hydroxide, the precipitate being dissolved and reprecipitated in order to remove the mesothorium completely. The precipitate was then dissolved in hydrochloric acid and allowed to stand for over a month so as to reach equilibrium between the radiothorium and thorium X.

An aliquot of the solution was then diluted to $150 \mathrm{cc}$. in a $250 \mathrm{cc}$. Erlenmeyer flask, some barium chloride added, and then the flask closed for several days to insure the presence in the solution of the maximum amounts of thorium B, C and D; $50 \mathrm{mg}$. each of lead and mercury as chlorides were then added, the acidity reduced by neutralization to the proper point

${ }^{1}$ Am. J. Sci., 22, 477 (1906).

2This JourNaL, 40, 1316 (1918). 